\title{
Abnormal auditory forward masking pattern in the brainstem response of individuals with Asperger syndrome
}

This article was published in the following Dove Press journal:

Neuropsychiatric Disease and Treatment

28 May 2010

Number of times this article has been viewed

\author{
Johan Källstrand' \\ Olle Olsson ${ }^{2}$ \\ Sara Fristedt Nehlstedt ${ }^{\prime}$ \\ Mia Ling Sköld' \\ Sören Nielzén ${ }^{2}$ \\ 'SensoDetect AB, Lund, Sweden; \\ ${ }^{2}$ Department of Clinical \\ Neuroscience, Section of Psychiatry, \\ Lund University, Lund, Sweden
}

\begin{abstract}
Abnormal auditory information processing has been reported in individuals with autism spectrum disorders (ASD). In the present study auditory processing was investigated by recording auditory brainstem responses (ABRs) elicited by forward masking in adults diagnosed with Asperger syndrome (AS). Sixteen AS subjects were included in the forward masking experiment and compared to three control groups consisting of healthy individuals $(n=16)$, schizophrenic patients $(n=16)$ and attention deficit hyperactivity disorder patients $(n=16)$, respectively, of matching age and gender. The results showed that the AS subjects exhibited abnormally low activity in the early part of their ABRs that distinctly separated them from the three control groups. Specifically, wave III amplitudes were significantly lower in the AS group than for all the control groups in the forward masking condition $(P<0.005)$, which was not the case in the baseline condition. Thus, electrophysiological measurements of ABRs to complex sound stimuli (eg, forward masking) may lead to a better understanding of the underlying neurophysiology of AS. Future studies may further point to specific ABR characteristics in AS individuals that separate them from individuals diagnosed with other neurodevelopmental diseases.
\end{abstract}

Keywords: asperger syndrome, auditory brainstem response, forward masking, psychoacoustics

\section{Introduction}

Asperger syndrome (AS) belongs to the autism spectrum disorders (ASD) and is characterized by stereotyped behavior and deficient social interaction and communication skills. ${ }^{1}$ ASD are, at least in part, regarded as neurodevelopmental disorders where certain frontotemporal neural circuitries and brainstem-cerebellar circuitries often are found to be dysfunctional. ${ }^{2}$

Auditory sensory processing has been shown to be deficient in ASD and AS.,4 Among the auditory symptoms reported is hypersensitivity to auditory stimulation and difficulties in filtering out environmental background noise. ${ }^{5-8}$ Although individuals with AS usually have normal language development, deficient semantic-pragmatic skills have been reported. ${ }^{9-11}$ The neural basis of language abnormalities in individuals with ASD has been widely studied using the recording of auditory event-related potentials (ERPs), which represent transient changes in the brain's electrical activity in response to certain auditory stimuli. Several studies have shown abnormal latencies and amplitudes of the early components (P1, N1, P2) of ERPs, suggesting abnormalities in basic sensory processing at an automatic, pre-attentional level. ${ }^{12}$ Furthermore, differences in higher level processing, such as novelty detection, sensory memory, discrimination of stimuli features, of auditory stimuli have also been widely observed. ${ }^{12-14}$ Several studies have shown reduced P3a and P3b responses,
Kyrkogatan 19, SE-222 22 Lund, Sweden

Tel $+46(0) 46 \quad$ I57904

Fax $+46(0) 46$ I3440 I

Email soren.nielzen@gmail.com 
suggesting impaired social orienting and novelty processing, and abnormalities in the mismatch negativity (MMN), reflecting encoding of acoustic change. ${ }^{12}$

Recently, Russo and colleagues for the first time demonstrated a subcortical involvement in deficient coding of pitch in children with ASD, presumably contributing to impairment in pragmatic, socially contextualized language. ${ }^{15}$ The study evaluated brainstem frequency-following responses (FFRs) evoked by speech syllables with ascending and descending pitch contours, showing abnormal brainstem processing in a subgroup of children with ASD.

The auditory brainstem response (ABR) is often affected in neuropsychiatric disorders. ${ }^{16}$ ABR reflects subcortical potentials evoked by brief auditory stimuli, and was first described by Jewett and Williston in $1971 .{ }^{17}$ The ABR consists of a sequence of seven positive peaks (waves I-VII) that normally occur within $10 \mathrm{~ms}$ following the onset of a stimulus. Waves I and II are produced by the auditory nerve, whereas the subsequent peaks are due to the combined electrical activity of nuclei at gradually higher levels of the ascending auditory pathway in the brainstem. Waves III and IV are believed to be generated in the cochlear nucleus and superior olivary complex (SOC), respectively, whereas wave $\mathrm{V}$ is thought to represent activity at the levels of lateral lemniscus and inferior colliculus. ${ }^{16,18}$ The ABR waveform provides information in terms of the latencies and amplitudes of these peaks. Analysis of the ABR wave patterns normally comprises measurements of interpeak latencies as well as peak amplitude ratios. ${ }^{19,20} \mathrm{ABR}$ is an objective method that does not require active patient participation. Therefore, use of the ABR technique is an applicable approach to investigate brainstem function in neuropsychiatric populations. Specifically, complex stimuli may reveal aberrations which may not be assessed by standard audiological ABR procedures. Complex stimuli (eg, forward masking) is therefore used in the present study to increase the possibility of detecting group differences. This may occur since AS patients are known to have deficits in complex processing. ${ }^{21}$

Previous studies by this group have shown that schizophrenic patients perceive sounds differently than nonschizophrenic comparison subjects, possibly partly reflecting abnormal functioning of the lower portion of the auditory pathway. ${ }^{22,23}$ More specifically, auditory masking has been shown to be aberrant for schizophrenic patients. ${ }^{22}$ Auditory forward masking refers to the reduced ability to detect a stimulus when preceded by a masking sound. ${ }^{24}$ The masking effect can be assessed as the amount of shift in latency and amplitude relative to the unmasked condition. Auditory forward masking has been shown to lead to prolonged latencies for waves III and V as well as reduced peak amplitude for wave III and increased wave V amplitude. ${ }^{25}$ Interestingly, speechin-noise perception difficulties have been observed for individuals diagnosed with AS, which in part are thought to reflect abnormal peripheral auditory processing. ${ }^{26}$ Thus, it is pertinent to further investigate auditory sensory dysfunctions in AS by means of auditory forward masking.

The aim of this study was to investigate auditory forward masking abilities of AS patients compared to matched healthy individuals as well as individuals of other neuropsychiatric conditions reportedly having sensory and perceptual dysfunctions such as schizophrenia and attention deficithyperactivity disorder (ADHD). Therefore, the subjects were chosen on the basis of diagnostic criteria of schizophrenia, ADHD, and AS according to the Diagnostic and Statistical Manual of Mental Disorders, 4th Edition (DSM-IV) as corroborated by senior psychiatrists only. No interest was focused on demographic or other background factors. Specifically, interpeak latencies and peak amplitudes in forward masking ABRs were investigated in this study. Furthermore, qualitative curve analysis was performed for comparison between the AS subjects and control groups.

\section{Methods \\ Subjects}

Sixteen AS patients (mean age ( \pm standard deviation $[\mathrm{SD}]$ ) $38.4 \pm 10.8$ years, age range $23-58 ; 11$ males, 5 females) were included in the study. The majority of the participants were males, reflecting the greater incidence of AS in males versus females. ${ }^{27}$ An equal number of reference subjects (mean age ( \pm SD) $38.3 \pm 12.4$ years, range 20-56; 11 males, 5 females) were chosen from a pool of mentally healthy individuals, to match age and gender of AS patients. As symptoms of ASD overlap with other psychiatric disorders, two groups (each group $\mathrm{n}=16$ ) consisting of schizophrenic (mean age ( \pm SD) $38.9 \pm 9.1$ years, range 24-56; 11 males, 5 females) and ADHD subjects (mean age $( \pm \mathrm{SD}) 37.4 \pm 10.9$ years, range $21-58$; 11 males, 5 females), respectively, were also included in the study. ${ }^{28,29}$ Patient groups had been diagnosed according to DSM-IV by senior psychiatrists. The diagnoses were established at least one year prior to testing. All subjects were tested to exclude hearing impairment. Hearing ability and control ABRs were investigated by an audiologist to exclude subjects with hearing disabilities from the study. As a consequence, one schizophrenic subject was excluded from the study. The AS, ADHD, and schizophrenic subjects had no neuropsychiatric co-morbidities. All schizophrenic subjects except one had neuroleptic treatment whereas 
reference and ADHD, subjects had no medication at the time of testing. Of the AS subjects, three were taking selective serotonin reuptake inhibitors and one was taking a selective norepinephrine reuptake inhibitor. There were no significant differences in the proportions of handedness between groups. Written informed consent was obtained from all subjects after the procedures had been explained fully. A formal consent was ascertained in accordance with the requirements of the ethical committee at the University of Lund, Sweden (document number 353/2006).

\section{Stimuli and apparatus}

A square-shaped click pulse was used as a probe for both the control ABR condition and the auditory forward masking setup. The probe had a duration time of 0.000136 seconds and a rise and fall time of 0.000023 seconds. The individual clicks of the stimulus train had an interstimulus interval (ISI) from onset to onset of 0.192 seconds. In the forward masking paradigm the square-shaped click pulse is preceded by a masker (Figure 1). A $1500 \mathrm{~Hz}$ low-pass filtered noise (Butterworth filter) was used as the masker. The duration of the masking noise was 0.015 seconds including the 0.004 seconds rise and fall time, and the gap between masker and target stimulus was 0.012 seconds. The time interval onset to onset of click in the forward masking setup was 0.192 seconds. The evoked potentials were recorded using the GN Otometrics' Chartr EP ABR recording equipment (GN Otometrics, Taastrup, Denmark). TTL trigger pulses coordinated the sweeps with the auditory stimuli. The click pulses were repeated until a total of 1024 accepted evoked potentials had been collected. Each ABR waveform represents an average of the responses to 1024 stimulus presentations. Aberrant activity, such as extremely high amplitudes due to extraordinary movements, was rejected using the standard setup GN Otometrics' Chartr software. The stimuli were presented to the subjects with an intensity level of $80 \mathrm{~dB}$ sound pressure level (SPL). Sound levels were calibrated using a Bruel and Kjaer 2203 sound level meter and Type 4152 artificial ear (Bruel and Kjaer S\&V Measurement, Naerum, Denmark).

All stimuli were constructed using MATLAB Signal Processing Toolbox (The MathWorks, Inc., Natick, MA, USA) and presented using a Denon DCD-685 compact disc player (Denon Electronics, Mahwah, NJ, USA). The output of the CD player was connected to TDH-50P headphones with Model 51 cushions (Telephonics, Farmingdale, NY, USA). Presentations were made binaurally with the stimuli in phase over headphones. The masking noise was kept constant at an intensity level of $70 \mathrm{~dB}$ SPL.

\section{Procedure}

All tests were performed in a quiet darkened room. Participants were comfortably seated in an armchair in a resting position. Surface electrodes were applied to the mastoid bone behind the left and right ear, with a ground electrode and a reference electrode placed on the vertex and forehead, respectively. Absolute impedances and interelectrode impedance were measured before and after the experiments to verify that electrode contact was maintained (below $5000 \Omega$ ). The subjects were instructed to relax with their eyes closed and were permitted to fall asleep. The test required no active participation other than being subjected to sound stimulation. Before the test session, subjects were verbally informed of the nature of the experiments. The click sounds were presented to the subjects beforehand to make them acquainted with stimuli. The subjects were tested one at a time and the duration of the testing procedure was 40 minutes.

\section{Data analysis}

All ABR waveforms were analyzed using Chartr GN software. A qualitative approach was taken as an audiologist

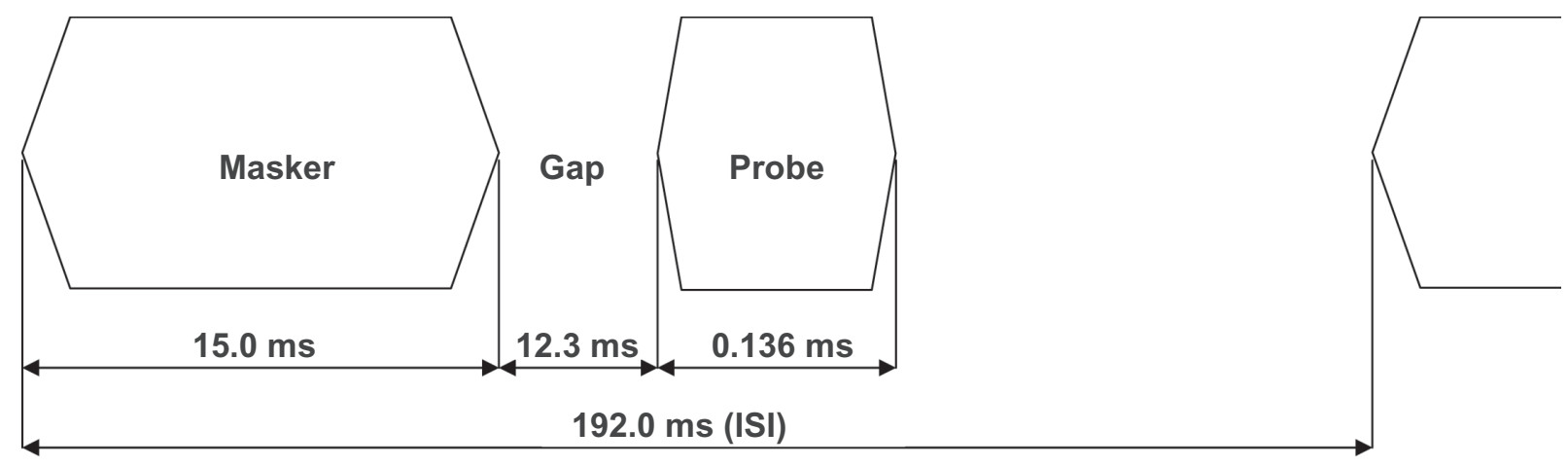

Figure I Graphical representation of the forward masking stimulus. 
and a physician visually investigated all ABR waveforms for identification of dominant peaks in specific regions. ABR waveforms were analyzed separately for each ear. Interpeak latencies and peak amplitudes were retrieved and transferred into Microsoft Excel (Microsoft Corp, Redmond, WA, USA) for further statistical analysis. Latency was measured from the onset of the stimulus to the peak of a given wave. Amplitude was measured from the positive peak of a given wave to the bottom of the following trough. In order to investigate masking effects, latency changes were expressed as amount of shift relative to the latency in the unmasked condition and amplitude changes were expressed as a percentage change from the unmasked condition. Following the measurements, the I/III, I/V and III/V peak amplitude ratios in the forward masking condition were calculated. The Mann-Whitney $U$ test was used for nonparametric group comparisons between AS patients and reference groups (Minitab, Sydney, NSW, Australia).

\section{Results}

The aim of the study was to examine ABR forward masking patterns of AS individuals compared to control subjects. Baseline condition consisted of repeated stimulation with a square-shaped click pulse. Analysis of latencies and peak amplitudes of the ABR waveforms in the baseline condition showed significant differences of peak III amplitudes between the AS and schizophrenia groups $(P=0.012)$ and between the AS and ADHD groups $(P=0.009)$. Transmission of basic auditory click stimulus was however not significantly altered in the group of AS subjects as compared to matched healthy controls.

When a masker was introduced before the square-shaped click pulse (Figure 1), forward masking effects such as tendencies of prolonged waves III and V latencies and reduced peak amplitude for wave III were seen in all groups (data not shown). No significant group differences were seen in masking effects, as measured by percentual amplitude and latency shifts between identified peaks in the two conditions (data not shown).

In between-group comparison of ABR waveforms in the forward masking condition showed that wave III amplitudes were significantly lower in AS individuals than in the control groups. This was statistically significant for AS individuals (Table 1$)$ versus healthy individuals $(P=0.002)$, ADHD subjects $(P=0.001)$ and schizophrenic subjects $(P=0.002)$. No significant differences regarding other peak amplitudes and latencies were found between AS subjects and the control groups in forward masking ABR waveforms.
Table I Peak III amplitude $(\mu \mathrm{V})$ of right side forward masking ABRs for subjects with Asperger syndrome and matched control groups

\begin{tabular}{|c|c|c|c|c|}
\hline & $\begin{array}{l}\text { Controls } \\
(n=16)\end{array}$ & $(n=16)$ & $\begin{array}{l}\text { Schizophrenia } \\
(n=16)\end{array}$ & $\begin{array}{l}\text { Asperger } \\
\text { syndrome } \\
(n=16)\end{array}$ \\
\hline $\begin{array}{l}\text { Amplitude } \\
\text { peak III } \\
(\text { mean } \pm S D)\end{array}$ & $0.33 \pm 0.16$ & $0.39 \pm 0.22$ & $0.42 \pm 0.28$ & $0.17 \pm 0.10$ \\
\hline$P$ value & $<0.005$ & $<0.005$ & $<0.005$ & - \\
\hline
\end{tabular}

Abbreviations: $S D$, standard deviation; $A B R s$, auditory brainstem responses; $A D H D$, attention deficit hyperactivity disorder.

Figure 2 shows the averaged right ear ABR waveforms of males without diagnosis $(n=5$, mean age 30.0 years, range 26-33) and with AS diagnosis $(n=5$, mean age 29.6 years, range 26-34), respectively, in response to forward masking. Homogenous groups were chosen to reduce the effects of age and sex in averaged ABR waveforms. Prolonged latencies could consistently be noted in the averaged ABR waveforms of the AS subjects compared to those of healthy individuals. Generally, a tendency of reduced wave amplitudes was observed in the averaged ABRs of AS patients, as compared to that of healthy individuals. Furthermore, the balance between the initial peaks was slightly altered.

The wave amplitude ratios I/III, I/V, III/V in the forward masking paradigm were investigated and analyzed using the Mann-Whitney $U$ test. No statistically significant differences of these amplitude ratios were found between AS subjects and the control groups. Wave I amplitudes were noted to be of high variability in all investigated groups, which consequently led to high variability in ratios involving this peak amplitude.

There was a general trend of similar left side differences that however did not reach statistically significant levels (data not shown).

\section{Discussion}

The present study was aimed at determining whether AS individuals and control subjects differ in their ABR patterns in response to forward masking. Previous studies have shown that AS patients as well as learning-disabled individuals, who also have auditory processing deficits, have normal ABRs to click stimuli, in contrast to more complex sounds eg, speech stimuli. ${ }^{15,30-33}$ Thus, our null findings regarding statistically significant differences between AS subjects and matched healthy controls in response to the square-shaped click pulse were not unexpected. Furthermore, this indicates that the basic auditory functioning is not significantly disturbed in 


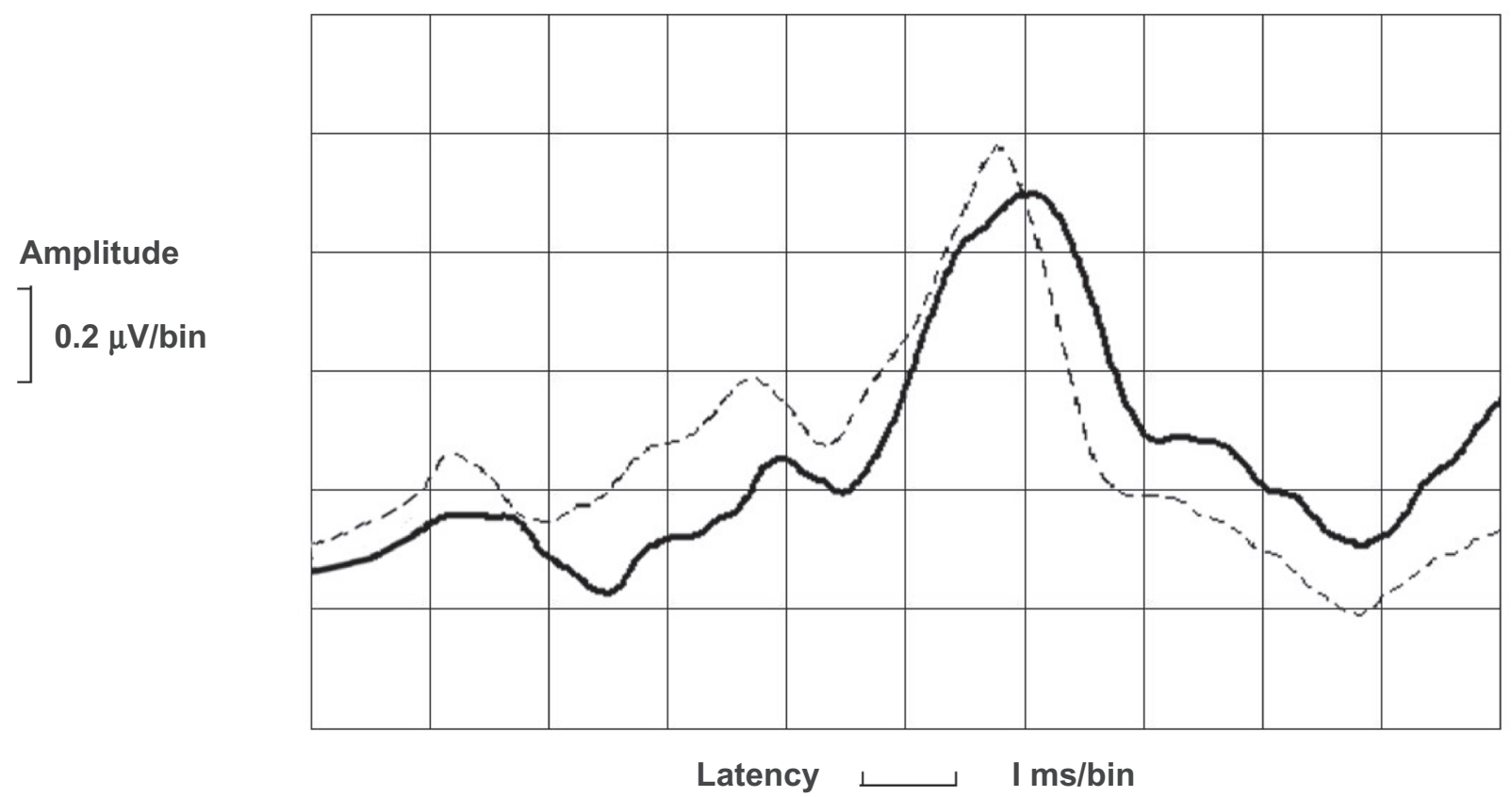

Figure 2 Averaged ABRs with forward masking stimulus for male subjects with Asperger syndrome ( $n=5$; soild line) and matched healthy controls ( $n=5$; dotted line).

AS patients. The significant wave III amplitude differences in the baseline condition between the AS subjects and the schizophrenic and ADHD groups, respectively, may reflect specific pathologies for mentioned groups.

As previously described, forward masking increases wave III and wave V latencies and leads to amplitude reduction of wave III, a trend which was observed in all groups. ${ }^{25}$ A tendency of ABR waveform abnormalities, such as prolongation of wave I latency, was observed in all patient groups, but not seen in healthy individuals (data not shown). However, forward masking revealed an abnormal pattern in the early part of the ABR of AS patients that significantly separated them from all control groups. Specifically, the lowered peak III amplitude in the forward masking paradigm separated AS subjects from both psychiatrically healthy matched controls and individuals diagnosed with schizophrenia or ADHD. The separation was statistically significant although a few AS subjects did not show the differentiating characteristics. The fact that not all AS subjects showed this abnormality might be explained by the diagnostic heterogeneity in AS individuals. However, the abnormalities were observed for the majority of the AS subjects, contrasting with the findings of other studies where deficits were only observed for a subgroup of AS patients. . $^{15,34,35}$

In forward masking, the masker precedes the target stimulus and the threshold of the target sound is thereby elevated by the masker. The masking effect generally increases with a shorter time interval between the masker and target stimulus, longer masker duration and when the masker and target stimulus frequencies are close together. ${ }^{36-38}$ Forward masking has been suggested to play a role in echo suppression, thereby enabling listeners to determine the true direction of the original sound source, despite the presence of echoes ${ }^{39}$ It is well known that ASD/AS individuals have difficulties separating competing sound sources and have reduced understanding of speech in a noisy environment although their basic sensory sound processing capacity has been claimed to be largely intact. ${ }^{6,26,30,40}$ However, auditory abnormalities in the low-level auditory processing in AS individuals have been described although the neural basis of these deficits is not well understood..$^{41}$ The auditory brainstem has been investigated in a number of studies of ASD patients. ${ }^{18}$ However, the majority of studies have focused on simple sounds in a small number of subjects, and the reported results are inconsistent. Rosenhall and colleagues reported in an extensive study that the majority of a group consisting of 101 children and young adults with mild autism showed abnormalities in the auditory brainstem, supporting the findings of previous studies suggesting that brainstem dysfunction is involved in ASD. . $^{34,35,42}$ Furthermore, magnetic resonance imaging revealed reduced brainstem size in ASD children and adults. ${ }^{43,44}$ Recently, Russo and colleagues demonstrated deficient processing of speech-evoked brainstem responses in a subset of children with ASD, suggesting that the brainstem is implicated in the processing of complex sounds. ${ }^{15}$ 
In the present study, the ABR of AS patients revealed an abnormal pattern in the early part of the ABR, corresponding to peripheral structures in the brainstem and in the auditory nerve. Wave I is produced by the auditory nerve, whereas wave III is thought to be generated in the cochlear nucleus or at the level of the superior olivary complex (SOC). ${ }^{16,45}$ Consequently, the abnormal response to forward masking in AS patients as measured by the significantly decreased peak III amplitude may suggest that electrical activity at the levels of the cochlear nucleus and/or superior olivary complex is decreased in AS patients. Indeed, forward masking has been shown to exert its effect on both the auditory nerve fiber and dorsal neurons in the cochlear nuclear complex. ${ }^{39,46}$ The medial olivocochlear (MOC) system, localized in the vicinity of SOC, is assumed to be involved in complex sound processing and may be involved in filtering ascending auditory input via efferent feedback from higher auditory nuclei and the auditory cortex..$^{47-50}$ The MOC system has been shown to suppress the response of the cochlea to concurrent noise thereby improving signal-to-noise detection. ${ }^{51}$ The MOC bundle synapses directly on the outer hair cells $(\mathrm{OHCs})$ that are involved in the amplification of cochlear vibrations in response to sound. Activation of the MOC system modulates the OHCs by decreasing their amplification effect. ${ }^{52}$ Interestingly it has been hypothesized that the MOC system may be dysfunctional in ASD individuals. ${ }^{53}$ Thus, a deficiency of the MOC system may contribute to the abnormalities in the ABR of AS individuals shown in our study.

The observed result of lower wave III amplitude in the forward masking condition of AS individuals was only statistically significant for results obtained from the right side. This indicates right ear dominance, which is in concordance with the general finding of lateral asymmetry in hearing sensitivity, as the right ear has been shown to have a lower average threshold than the left ear. ${ }^{54}$ Interestingly, the MOC system has been shown to have greater influence on the right ear..$^{55} \mathrm{~A}$ lateralization effect involving the MOC system could reflect lateralization of cochlear mechanisms. ${ }^{56}$ It has been suggested that the brainstem may be involved in the resulting lateralization deficits which could affect both afferent and efferent auditory pathways. ${ }^{56}$

Auditory processing involves the brainstem and subcortical structures together with cortical networks. The afferent pathway is not just limited to straightforward bottom-up signaling, it is also collaterally influenced, and investigated structures might be affected by neurons at higher levels of the auditory pathway. It has been suggested that auditory stimulus processing early in the auditory pathway may influence later cortical events and higher cognitive processes can modify neural activity via top-down processing mediated via descending pathway. ${ }^{57}$ Furthermore, the cortex has been indicated to have a possible role in forward masking. ${ }^{58}$ Thus, the abnormal ABR to forward masking in AS individuals may partly involve efferent cortical influence. However, the fact that this study is based on a passive listening task, wherein the subjects were not required to actively listen to the probe, suggests that pre-attentive brainstem deficits play a key role.

A number of studies have focused on neuronal development and functional connectivity in ASD. In ASD children there is evidence of a normal brain size at birth followed by overgrowth during the first 6-14 months, coinciding with a period of, abundant synaptogenesis, myelination and elaboration of dendritic and axonal processes, suggesting overgrowth of neural connections and a lack of selective elimination of neural processes. ${ }^{59}$ Increased volumes of cerebellar and cerebral white matter have been reported in several studies, indicating differences in axonal density, myelination abnormalities or abnormal glial cell proliferation. ${ }^{60,61}$ Furthermore, the neural connectivity is suggested to be abnormal in ASD individuals. ${ }^{21}$ Specifically, longrange connectivity between functional brain regions may be abnormally weak whereas overgrowth of local projections has been reported. ${ }^{62,63}$ Deficits in functional connectivity may not account for the findings of this study, although such effects cannot be fully excluded.

The results of this study implicate deficits in auditory forward masking of AS individuals, suggesting a role of the early brainstem in the pathology of the disease. There were, however, several limitations in the present study, one of them being that larger samples of AS patients will be needed in a future study to further support this finding. Another issue to be considered is that age and gender influences the ABR characteristics. ${ }^{64}$ It is well known that males consistently show longer ABR latencies than females. In this study only minor latency differences were discovered between male and female AS patients and due to the small number of female participants no statistical analysis could be done. Neither could the effects of medication be assessed this time due to the small number of participants. However, earlier studies in this group have shown that medication effects on the ABR are much smaller than the effect of an unquestionable neuropsychiatric disease such as schizophrenia or AS (unpublished results). In a future study, it would be interesting to investigate whether the abnormalities in the forward masking ABR waveforms of AS patients can be correlated with other parameters such 
as language impairment, using rating scales for the subjects. Control groups diagnosed with additional neuropsychiatric disorders could also be included in a future study to further investigate the specificity in the ABR patterns of AS patients compared to patients diagnosed with other neuropsychiatric disorders.

\section{Conclusions}

Auditory brainstem processing of forward masking showed clear differences in ABR waveforms of AS patients when compared to the other control groups of this study. Aberrant early brainstem auditory network functioning thus exists in these patients and it is possible to demonstrate it by means of ABR. The high level of statistical significance regarding the AS patients versus all control groups indicates a specificity of discrimination. This fact may bear a great importance for further studies with the aim to use ABR for diagnostic purposes and/or in the process of controlling therapeutic efforts in neuropsychiatric states.

\section{Acknowledgments}

We would like to thank Åsa Sandstedt Eriksson, Agneta Julinder and Pernilla Remsö at the NPU unit in Linköping, Sweden for their contribution to this research by performing tests and collecting data. We would also like to thank Professor Anders Forsman for valuable discussions. This research was supported by the Sjöbring foundation at the Department of Neuroscience, University of Lund.

\section{Disclosures}

Authors Johan Källstrand, Olle Olsson, and Sara Fristedt Nehlstedt are employees of, and hold stock in, SensoDetectAB. Author Mia Ling Sköld is an employee of SensoDetect AB. Sören Nielzén, Assistant Professor of Psychiatry at the University of Lund, holds stock in SensoDetect AB although not employed by the company.

\section{References}

1. American Psychiatric Association. Diagnostic and Statistical Manual of Mental Disorders. Fourth edition. Washington, DC: American Psychiatric Association; 1994.

2. Gillberg C. Neurodevelopmental processes and psychological functioning in autism. Dev Psychopathol. 1999;11(3):567-587.

3. Jones CR, Happe F, Baird G, et al. Auditory discrimination and auditory sensory behaviours in autism spectrum disorders. Neuropsychologia. 2009;47(13):2850-2858.

4. Dunn W, Myles BS, Orr S. Sensory processing issues associated with Asperger syndrome: a preliminary investigation. Am J Occup Ther. 2002;56(1):97-102.

5. Bettison $\mathrm{S}$. The long-term effects of auditory training on children with autism. J Autism Dev Disord. 1996;26(3):361-374.
6. Teder-Sälejärvi WA, Pierce KL, Courchesne E, Hillyard SA. Auditory spatial localization and attention deficits in autistic adults. Brain Res Cogn Brain Res. 2005;23(2-3):221-234.

7. Rosenhall U, Nordin V, Sandström M, Ahlsén G, Gillberg C. Autism and hearing loss. J Autism Dev Disord. 1999;29(5):349-357.

8. Lepistö T, Kuitunen A, Sussman E, et al. Auditory stream segregation in children with Asperger syndrome. Biol Psychol. 2009;82(3):301-307.

9. Kerbel D, Grunwell P. A study of idiom comprehension in children with semantic-pragmatic difficulties. Part II: Between-groups results and discussion. Int J Lang Commun Disord. 1998;33(1):23-44.

10. McCann J, Peppe S. Prosody in autism spectrum disorders: a critical review. Int J Lang Commun Disord. 2003;38(4):325-350.

11. Saalasti S, Lepistö T, Toppila E, et al. Language abilities of children with Asperger syndrome. J Autism Dev Disord. 2008;38(8):1574-1580.

12. Jeste SS, Nelson CA 3rd. Event related potentials in the understanding of autism spectrum disorders: an analytical review. J Autism Dev Disord. 2009;39(3):495-510.

13. Lepistö T, Nieminen-von Wendt T, von Wendt L, Näätänen R, Kujala T. Auditory cortical change detection in adults with Asperger syndrome. Neurosci Lett. 6 2007;414(2):136-140.

14. Lepistö T, Silokallio S, Nieminen-von Wendt T, Alku P, Näätänen R, Kujala T. Auditory perception and attention as reflected by the brain event-related potentials in children with Asperger syndrome. Clin Neurophysiol. 2006;117(10):2161-2171.

15. Russo NM, Skoe E, Trommer B, et al. Deficient brainstem encoding of pitch in children with autism spectrum disorders. Clin Neurophysiol. 2008;119(8):1720-1731.

16. Wilkinson AR, Jiang ZD. Brainstem auditory evoked response in neonatal neurology. Semin Fetal Neonatal Med. 2006;11(6):444-451.

17. Jewett DL, Williston JS. Auditory-evoked far fields averaged from the scalp of humans. Brain. 1971;94(4):681-696.

18. Klin A. Auditory brainstem responses in autism: brainstem dysfunction or peripheral hearing loss? J Autism Dev Disord. 1993;23(1):15-35.

19. Sand T. Statistical properties of ABR amplitudes and latencies. Implications for computation of reference limits and relation to click phase. Scand Audiol. 1990;19(3):131-137.

20. Musiek FE, Lee WW. The auditory brain stem response in patients with brain stem or cochlear pathology. Ear Hear. 1995;16(6):631-636.

21. Minshew NJ, Williams DL. The new neurobiology of autism: cortex, connectivity, and neuronal organization. Arch Neurol. 2007;64(7): 945-950.

22. Källstrand J, Montnémery P, Nielzén S, Olsson O. Auditory masking experiments in schizophrenia. Psychiatry Res. 2002;113(1-2):115-125.

23. Nielzén S, Olsson O, Källstrand J, Nehlstedt S. Aberrant brain stem function in schizophrenia. Eur Psychiatry. 2008;23 Suppl 2:S135.

24. Kramer SJ, Teas DC. Forward masking of auditory nerve (N1) and brainstem (wave V) responses in humans. JAcoust Soc Am. 1982;72(3): 795-803.

25. Ananthanarayan AK, Gerken GM. Response enhancement and reduction of the auditory brain-stem response in a forward-masking paradigm. Electroencephalogr Clin Neurophysiol. 1987;66(4):427-439.

26. Alcántara JI, Weisblatt EJ, Moore BC, Bolton PF. Speech-in-noise perception in high-functioning individuals with autism or Asperger's syndrome. J Child Psychol Psychiatry. 2004;45(6):1107-1114.

27. Roy M, Dillo W, Emrich HM, Ohlmeier MD. Asperger's syndrome in adulthood. Dtsch Arztebl Int. 2009;106(5):59-64.

28. Nylander L, Lugnegård T, Unenge Hallerbäck M. Autism spectrum disorders and schizophrenia spectrum disorders in adults - is there a connection? A literature review and some suggestions for future clinical research. Clin Neuropsych. 2008;5(1):43-54.

29. Stahlberg O, Soderstrom H, Rastam M, Gillberg C. Bipolar disorder, schizophrenia, and other psychotic disorders in adults with childhood onset $\mathrm{AD} / \mathrm{HD}$ and/or autism spectrum disorders. J Neural Transm. 2004;111(7):891-902.

30. Russo N, Nicol T, Trommer B, Zecker S, Kraus N. Brainstem transcription of speech is disrupted in children with autism spectrum disorders. Dev Sci. 2009;12(4):557-567. 
31. Banai K, Nicol T, Zecker SG, Kraus N. Brainstem timing: implications for cortical processing and literacy. J Neurosci. 2005;25(43): 9850-9857.

32. Song JH, Banai K, Russo NM, Kraus N. On the relationship between speech- and nonspeech-evoked auditory brainstem responses. Audiol Neurootol. 2006;11(4):233-241.

33. Johnson KL, Nicol TG, Zecker SG, Kraus N. Auditory brainstem correlates of perceptual timing deficits. $J$ Cogn Neurosci. 2007;19(3): 376-385.

34. Maziade M, Merette C, Cayer M, et al. Prolongation of brainstem auditory-evoked responses in autistic probands and their unaffected relatives. Arch Gen Psychiatry. 2000;57(11):1077-1083.

35. Rosenhall U, Nordin V, Brantberg K, Gillberg C. Autism and auditory brain stem responses. Ear Hear. 2003;24(3):206-214.

36. Elliott LL. Backward and forward masking of probe tones of different frequencies. J Acoust Soc Am. 1962;34(8):1116-1117.

37. Weber DL. Do off-frequency simultaneous maskers suppress the signal? J Acoust Soc Am. 1983;73(3):887-893.

38. Carlyon RP. The development and decline of forward masking. Hear Res. 1988;32(1):65-79.

39. Kaltenbach JA, Meleca RJ, Falzarano PR, Myers SF, Simpson TH. Forward masking properties of neurons in the dorsal cochlear nucleus: possible role in the process of echo suppression. Hear Res. 1993;67(1-2):35-44.

40. Ceponiene R, Lepistö T, Shestakova A, et al. Speech-sound-selective auditory impairment in children with autism: they can perceive but do not attend. Proc Natl Acad Sci U SA. 2003;100(9):5567-5572.

41. Korpilahti P, Jansson-Verkasalo E, Mattila ML, et al. Processing of affective speech prosody is impaired in Asperger syndrome. J Autism Dev Disord. 2007;37(8):1539-1549.

42. Wong V, Wong SN. Brainstem auditory evoked potential study in children with autistic disorder. J Autism Dev Disord. 1991;21(3):329-340.

43. Hashimoto T, Tayama M, Miyazaki M, et al. Brainstem involvement in high functioning autistic children. Acta Neurol Scand. 1993; 88(2): $123-128$.

44. Hashimoto T, Tayama M, Murakawa K, et al. Development of the brainstem and cerebellum in autistic patients. J Autism Dev Disord. $1995 ; 25(1): 1-18$

45. Koprdova I, Ciganek L. Pathology of wave III of brainstem auditory evoked potentials (BAEPs). Acta Physiol Hung. 1992;79(3):323-330.

46. Relkin EM, Turner CW. A reexamination of forward masking in the auditory nerve. J Acoust Soc Am. 1988;84(2):584-591.

47. Di Girolamo S, Napolitano B, Alessandrini M, Bruno E. Experimental and clinical aspects of the efferent auditory system. Acta Neurochir Suppl. 2007;97(Pt 2):419-424.

48. Thompson AM, Thompson GC. Relationship of descending inferior colliculus projections to olivocochlear neurons. J Comp Neurol. $1993 ; 335(3): 402-412$
49. Vetter DE, Saldana E, Mugnaini E. Input from the inferior colliculus to medial olivocochlear neurons in the rat: a double label study with PHA-L and cholera toxin. Hear Res. 1993;70(2):173-186.

50. Khalfa S, Bougeard R, Morand N, et al. Evidence of peripheral auditory activity modulation by the auditory cortex in humans. Neuroscience. 2001;104(2):347-358.

51. Winslow RL, Sachs MB. Effect of electrical stimulation of the crossed olivocochlear bundle on auditory nerve response to tones in noise. J Neurophysiol. 1987;57(4):1002-1021.

52. Liberman MC, Guinan JJ Jr. Feedback control of the auditory periphery: anti-masking effects of middle ear muscles vs olivocochlear efferents. J Commun Disord. 1998;31(6):471-482.

53. Collet L, Roge B, Descouens D, Moron P, Duverdy F, Urgell H. Objective auditory dysfunction in infantile autism. Lancet. 1993;342(8876): 923-924.

54. Pirilä T, Jounio-Ervasti K, Sorri M. Left-right asymmetries in hearing threshold levels in three age groups of a random population. Audiology. 1992;31(3):150-161.

55. Khalfa S, Collet L. Functional asymmetry of medial olivocochlear system in humans. Towards a peripheral auditory lateralization. Neuroreport. 1996;7(5):993-996.

56. Khalfa S, Bruneau N, Roge B, et al. Peripheral auditory asymmetry in infantile autism. Eur J Neurosci. 2001;13(3):628-632.

57. Galbraith GC, Gutterson RP, Levy DS, Mussey JL, Sabatasso FA, Wasserman RI. Correlated brain stem and cortical evoked responses to auditory tone change. Neuroreport. 2004;15(17):2613-2616.

58. Calford MB, Semple MN. Monaural inhibition in cat auditory cortex. J Neurophysiol. 1995;73(5):1876-1891.

59. Courchesne E, Carper R, Akshoomoff N. Evidence of brain overgrowth in the first year of life in autism. JAMA. 2003;290(3):337-344.

60. Herbert MR, Ziegler DA, Deutsch CK, et al. Dissociations of cerebral cortex, subcortical and cerebral white matter volumes in autistic boys. Brain. 2003;126(Pt 5):1182-1192.

61. Courchesne E, Karns CM, Davis HR, et al. Unusual brain growth patterns in early life in patients with autistic disorder: an MRI study. Neurology. 2001;57(2):245-254.

62. Belmonte MK, Allen G, Beckel-Mitchener A, Boulanger LM, Carper RA, Webb SJ. Autism and abnormal development of brain connectivity. J Neurosci. 2004;24(42):9228-9231.

63. Herbert MR, Ziegler DA, Makris N, et al. Localization of white matter volume increase in autism and developmental language disorder. Ann Neurol. 2004;55(4):530-540.

64. Allison T, Wood CC, Goff WR. Brain stem auditory, pattern-reversal visual, and short-latency somatosensory evoked potentials: latencies in relation to age, sex, and brain and body size. Electroencephalogr Clin Neurophysiol. 1983;55(6):619-636.
Neuropsychiatric Disease and Treatment

\section{Publish your work in this journal}

Neuropsychiatric Disease and Treatment is an international, peerreviewed journal of clinical therapeutics and pharmacology focusing on concise rapid reporting of clinical or pre-clinical studies on a range of neuropsychiatric and neurological disorders. This journal is indexed on PubMed Central, the 'PsycINFO' database and CAS, and is the official
Dovepress

journal of The International Neuropsychiatric Association (INA). The manuscript management system is completely online and includes a very quick and fair peer-review system, which is all easy to use. Visit http://www.dovepress.com/testimonials.php to read real quotes from published authors. 